\title{
ENERGIA METABOLIZÁVEL PARA SUÍNOS IMUNOCASTRADOS EM TERMINAÇÃO
}

(Metabolizable energy to imunocastreted barrows on termination phase)

\author{
Edison Torres da Silva Júnior¹, Rita da Trindade Ribeiro Nobre Soares¹, Juliano Pelição Molino², Fábio \\ da Costa Henry ${ }^{1}$, José Geraldo Vargas Junior ${ }^{3}$, Rodrigo Fortunato de Oliveira ${ }^{1}$
}

\begin{abstract}
1 Universidade Estadual do Norte Fluminense Darcy Ribeiro (UENF), Campos dos Goytacazes, Rio de Janeiro, Brasil; ${ }^{2}$ Instituto Federal do Espírito Santo (IFES), Montanha, Espírito Santo, Brasil; 3Universidade Federal do Espírito Santo (UFES), Vitória, Espírito Santo, Brasil.
\end{abstract}

*Corresponding author: fortunatorodrigo@ymail.com

RESUMO - Objetivou-se estimar a exigência de energia metabolizável (EM) para suínos machos imunocastrados na fase de terminação. O experimento foi conduzido na Universidade Estadual Norte Fluminense Darcy Ribeiro. Foram utilizados 60 suínos machos distribuídos em delineamento de blocos casualizados, sendo 50 suínos imunocastrados - IM (tratamento 1 a 5) e 10 castrados cirurgicamente - CC (tratamento 3b). Os tratamentos diferiram quanto ao nível de EM utilizada, sendo T1 - 3030, T2 3130, T3a - 3230, T3b - 3230, T4 - 3330 e T5 - $3430 \mathrm{Kcal}$ de EM/Kg. Foram avaliadas características de desempenho em diferentes períodos ( $\mathrm{A}$ - intervalo entre a primeira e a segunda imunização, B - intervalo entre a segunda imunização e o abate e $T$ - período total, da primeira imunização ao abate) e de carcaça, além de análise sensorial da carne dos suínos. Os dados médios obtidos nos diferentes níveis de energia foram submetidos à análise de variância e análise de regressão linear em nível de $5 \%$ de significância. $A$ análise foi realizada com o auxílio do programa de análises estatísticas SAEG. Foi observado efeito linear crescente $(P<0,05)$ para consumo de ração diário, ganho de peso diário, consumo diário de energia e consumo diário de lisina em diferentes períodos. Houve resultados significativos $(P<0,01)$ para consumo diário de energia e consumo de lisina em diferentes períodos. Não houve diferença $(P>0,05)$ entre as características de carcaça avaliadas. Na análise sensorial, embora os avaliadores tenham detectado diferenças no odor das carnes de suínos CC e IM, tais diferenças não foram devidas ao odor sexual. Não foi possível estimar o nível de energia metabolizável para a categoria de suínos imunocastrados em fase de terminação.

Palavras-chave: androsterona; castração cirúrgica; escatol; imunocastração; nutrição.

ABSTRACT - The objective was to estimate the metabolizable energy requirement (ME) for male pigs immunocastrated in the finishing phase. The experiment was conducted at 
the Universidade Estadual Norte Fluminense Darcy Ribeiro. 60 male pigs were used in a randomized block design, with 50 immunocastrated pigs - IM (treatment 1 to 5) and 10 surgically castrated - CC (treatment $3 \mathrm{~b}$ ). The treatments differed according to the level of MS used, being T1 - 3030, T2 - 3130, T3a - 3230, T3b - 3230, T4 - 3330 and T5 - 3430 Kcal of EM / Kg. Performance characteristics were evaluated in different periods (A interval between the first and second immunizations, B - interval between the second immunization and slaughter and $T$ - total period, from the first immunization to slaughter) and carcass, in addition to sensory analysis pig meat. The average data obtained at the different energy levels were subjected to analysis of variance and linear regression analysis at a level of significance of $5 \%$. The analysis was performed with the aid of the SAEG statistical analysis program. Increasing linear effect $(P<0.05)$ was observed for daily feed intake, daily weight gain, daily energy consumption and daily lysine consumption in different periods. There were significant results $(P<0.01)$ for daily energy consumption and lysine consumption in different periods. There was no difference $(P>0.05)$ between the carcass characteristics evaluated. In the sensory analysis, although the evaluators detected differences in the odor of pork from CC and IM pigs, such differences were not due to sexual odor. It was not possible to estimate the level of metabolizable energy for the category of immunocastrated pigs in the finishing phase.

Key words: androsterone; immunocastration; nutrition; skatole; surgical castration.

\section{INTRODUÇÃO}

A castração cirúrgica (gonadectomia) na suinocultura durante muito tempo foi realizada também com a justificativa de que os animais castrados apresentam maior deposição de gordura, manejo facilitado e menor incidência de danos nas carcaças por disputas hierárquicas no rebanho. Tudo isso combinado ao maior peso ao abate tornou a castração cirúrgica atrativa aos produtores e consumidores de produtos suinícolas.

No cenário atual da produção de carne suína, as carcaças com grande percentual de gordura já não são bem aceitas por parte do consumidor, que exige cada vez mais que a carne seja mais magra e de melhor qualidade. Além disso, existe grande tendência de maior aceitabilidade de produtos de origem animal quando estes condizem os preceitos de bem-estar na produção. A forte pressão exercida por países da União Europeia conduz as empresas de processamento de produtos cárneos e suinocultores a adotarem padrões produtivos mais elevados e tecnificados, levando em consideração não só o potencial de produção (baixos custos de produção por elevada margem de lucro) como também as inter-relações entre animais, instalações e manejo, caminhando para o 
bem-estar animal. Essa pressão exercida, que beira até mesmo os conceitos de ética, tem colaborado para o desuso da castração cirúrgica tida como tradicional, sem qualquer procedimento anestésico.

Neste contexto, o uso de animais inteiros se justificaria, já que apresenta maior deposição de carne magra quando comparados aos animais castrados, melhor conversão alimentar e ganho de peso semelhante. A utilização de suínos machos inteiros é alvo de restrições nos sistemas de produção, acompanhada também de certa rejeição por parte dos consumidores que relacionam a figura do animal inteiro com o odor e/ou sabor característico e indesejável, depositados na gordura dos animais que atingem a maturidade sexual. Diante de pressões dos consumidores em relação ao bem-estar dos animais e das preferências quanto às características da carne, novas tecnologias têm sido adotadas para evitar a castração cirúrgica e viabilizar a produção de suínos machos inteiros (Bogoni et al., 2015).

Os métodos de castração não-cirúrgica envolvem principalmente a imunocastração, tratamento hormonal, destruição do tecido testicular por compostos químicos à base de formaldeído, ácido acético, sal de prata ou zinco (Lopes e Silva, 2014). A utilização de andrógenos como terapia hormonal comumente utilizada em animais domésticos é mais eficaz que o uso de progestágenos, tornando-se uma alternativa, pois atua na liberação de $\mathrm{LH}$ e $\mathrm{FSH}$, diminuindo as concentrações de testosterona intratesticular e subsequente bloqueio da produção espermática (Lopes e Silva, 2014). A imunoesterilização, em que se enquadra a imunocastração, age de modo diferente da terapia hormonal, levando ao próprio organismo a produzir agentes (anticorpos) contra elementos necessários à espermatogênese (Lopes e Silva, 2014). Esses métodos provaram ser eficazes na esterilização dos animais, com comprometimento total ou parcial da produção de espermatozoides (Lopes e Silva, 2014), porém, em suínos não basta apenas o decréscimo ou eliminação das funções reprodutivas, se faz necessário utilizar de métodos que eliminem a produção de testosterona e androsterona.

Outros odores derivados da oxidação lipídica (ácidos graxos de cadeia curta, cetonas ou aldeídos) ou em processos de digestão intestinal (compostos fenólicos), também são sugeridos por alguns autores como contribuintes para o boar taint (Fischer et al., 2014). De forma adaptativa às exigências mercadológicas, produtores e pesquisadores vêm analisando alguns métodos de produção de suínos que previnam o boar taint, e dentre eles destaca-se atualmente a técnica de imunocastração. O uso da imunocastração surge como alternativa viável já que elimina os odores e permite o alcance de idade avançada, sem a necessidade do abate precoce, atingindo com isso o peso satisfatório. 
O uso de animais imunocastrados na atividade suinícola tende substancialmente a tornar a produção menos onerosa, já que os animais apresentarão melhor desempenho, aproveitando os efeitos anabólicos dos esteróides para deposição de carne, além de se evitar o sofrimento e diminuição da taxa de mortalidade, sobretudo na primeira semana, período em que normalmente se realiza a castração tradicional.

Os animais alimentam-se preferencialmente para suprir suas exigências em energia, portanto possibilita a afirmação de que a densidade energética das dietas seja um dos fatores, senão o mais importante, a ser determinado no momento em que se define o plano nutricional. A concentração de testosterona pode afetar a exigência e consumo de energia nesses animais, visto que este hormônio age na partição de nutrientes, basicamente mudando a prioridade dos tecidos favorecendo a deposição de tecido proteico em detrimento a de gordura.

Levando-se em consideração os custos com produção e disponibilização de ração na suinocultura pode representar em torno de $70 \%$ dos custos totais de produção, imperioso se faz considerar a possibilidade adotar arraçoamento mais eficiente, ou seja, estabelecer planos nutricionais visando menores excedentes possíveis seja em grãos, aminoácidos ou fontes e níveis energéticos baseados nas exigências de mantença e produção dos animais.

As possibilidades de melhorias no desempenho concomitantes ao bem-estar animal habilitam o produtor a resistir às pressões do mercado competitivo, principalmente o mercado exterior. A adequação dos níveis energéticos da dieta, em $\mathrm{Kcal} /$ dia, dessa nova categoria de suínos pode contribuir para a renovação nos sistemas atuais de criação, cuja grande maioria opta por animais castrados cirurgicamente, talvez pelo fato de não conhecer os benefícios da criação de imunocastrados ou pela mistificação dos odores desagradáveis na carne.

Objetivou-se estimar as recomendações nutricionais dos suínos imunocastrados na fase de terminação, baseando-se nas formulações de dietas com diferentes níveis de energia metabolizável para as diferentes categorias animais (castrados cirurgicamente e imunocastrados) a partir da primeira aplicação da vacina até o final da terminação.

\section{MATERIAL E METODOS}

O experimento foi conduzido nas instalações do Setor de Suinocultura do Laboratório de Zootecnia e Nutrição Animal do Centro de Ciências e Tecnologias Agropecuárias (LZNA/CCTA), da Universidade Estadual Norte Fluminense Darcy Ribeiro. Foram utilizados 60 suínos machos com aproximadamente $85 \pm 8,7 \mathrm{~kg}$ de peso vivo 
inicial e 112 dias de idade, sendo que 10 foram submetidos à gonadectomia (castração cirúrgica) e 50 foram submetidos à imunocastração.

Os animais foram distribuídos em um delineamento em blocos casualizados (DBC) com seis tratamentos e cinco repetições (blocos) com dois animais por unidade experimental. Foram utilizados seis tratamentos com diferentes níveis de energia metabolizável (EM): T1 - 3030 kcal EM/kg; T2 - 3130 kcal EM/kg; T3a - 3230 kcal EM/kg; T3b - $3230 \mathrm{kcal} \mathrm{EM/kg} \mathrm{T4} \mathrm{-} 3330 \mathrm{kcal} \mathrm{EM/kg} \mathrm{e} \mathrm{T5} \mathrm{-} 3430 \mathrm{kcal} \mathrm{EM/kg}$. O T3b teve como unidades experimentais animais castrados cirurgicamente aos 7 dias de idade, com nível de EM preconizado pela Tabela Brasileira de Aves e Suínos (Rostagno et al., 2017) e equiparado ao nível de energia do T3a.

Os animais imunocastrados receberam a primeira dose da vacina constituída de um análogo sintético incompleto do GnRh, conjugado à uma proteína carreadora, na dose de $2 \mathrm{~mL}$ e aplicação subcutânea, em duas aplicações, de acordo com o protocolo de aplicação (PFIZER). A primeira dose da vacina, que é a dose de sensibilização, foi aplicada no início do experimento (112 dias de idade) e a última dose foi aplicada 4 semanas depois (140 dias de idade). Em ambas as etapas de vacinação os animais foram pesados, visando estimar o peso vivo e ganho de peso dos animais antes e depois da aplicação.

Os animais foram alimentados duas vezes ao dia (8 e 16 horas) de modo a sobrar ração no cocho e com livre acesso a água. O consumo de ração e as sobras foram computados sempre no período da aplicação da segunda imunização (20 semanas) e ao final do experimento (abate com 24 semanas). Os animais foram pesados em três momentos (início do experimento, aplicação da segunda imunização e abate), utilizandose uma balança analógica. As características de desempenho avaliadas foram separadas em 3 períodos objetivando a observação na diferenciação no comportamento alimentar antes e após a segunda imunização: (período A - correspondente ao intervalo da primeira aplicação da vacina à segunda aplicação; período B - referente ao intervalo entre a segunda imunização ao abate; período $\mathrm{T}$ - caracterizando o período total experimental).

As rações experimentais (Tabela 1) foram baseadas em exigências nutricionais da Tabela Brasileira de Aves e Suínos de acordo com Rostagno et al. (2017). As rações experimentais são isonutritivas, porém não isocalóricas, formuladas com milho e farelo de soja, com adição de material inerte (areia lavada e peneirada) e óleo de soja de acordo com os níveis desejados de EM. 
Houve pesagem dos animais na véspera do abate (24 semanas de idade) para a determinação do peso vivo (PV) e após 12 horas de jejum com livre acesso a água os animais foram abatidos para determinar o peso da carcaça (PC). $\mathrm{O}$ abate

Tabela 1 - Composição nutricional e centesimal das rações experimentais ofertadas aos animais na fase de terminação.

\begin{tabular}{|c|c|c|c|c|c|c|}
\hline \multirow{2}{*}{ Ingrediente } & \multicolumn{6}{|c|}{ Nível de Energia Metabolizável (Kcal/Kg) } \\
\hline & 3030 & 3130 & 3230 & 3330 & 3430 & $3230 * 1$ \\
\hline Milho & 68,25 & 68,25 & 68,25 & 68,25 & 68,25 & 79,95 \\
\hline Farelo de Soja & 22,70 & 22,70 & 22,70 & 22,70 & 22,70 & 12,70 \\
\hline Lisina $\mathrm{HCL}$ & 0,37 & 0,37 & 0,37 & 0,37 & 0,37 & 0,27 \\
\hline L-Treonina & 0,154 & 0,154 & 0,154 & 0,154 & 0,154 & 0,064 \\
\hline DL-Metionina & 0,112 & 0,112 & 0,112 & 0,112 & 0,112 & 0,002 \\
\hline L-Triptofano & 0,017 & 0,017 & 0,017 & 0,017 & 0,017 & 0,012 \\
\hline Inerte & 4,300 & 3,040 & 1,800 & 0,600 & 0,010 & 1,200 \\
\hline Óleo de Soja & 0,100 & 1,360 & 2,600 & 3,800 & 5,400 & 1,800 \\
\hline Núcleo*2 & 4,000 & 4,000 & 4,000 & 4,000 & 4,000 & 4,000 \\
\hline \multicolumn{7}{|l|}{ Composição Calculada } \\
\hline Energia Metabolizável (Kcal/Kg) & 3030 & 3130 & 3230 & 3330 & 3430 & $3230 * 1$ \\
\hline Proteína Bruta (\%) & 16,24 & 16,24 & 16,24 & 16,24 & 16,24 & 12,38 \\
\hline Lisina (\%) & 1,000 & 1,000 & 1,000 & 1,000 & 1,000 & 0,692 \\
\hline Treonina Dig. (\%) & 0,670 & 0,670 & 0,670 & 0,670 & 0,670 & 0,463 \\
\hline Metionina + Cistina Dig. (\%) & 0,600 & 0,600 & 0,600 & 0,600 & 0,600 & 0,415 \\
\hline Metionina Dig. (\%) & 0,350 & 0,350 & 0,350 & 0,350 & 0,350 & 0,203 \\
\hline Cinzas & 6,840 & 5,608 & 4,393 & 3,217 & 2,623 & 3,419 \\
\hline Cálcio & 0,850 & 0,850 & 0,850 & 0,850 & 0,850 & 0,820 \\
\hline Fosforo Disponível (\%) & 0,300 & 0,300 & 0,300 & 0,300 & 0,300 & 0,288 \\
\hline Triptofano Dig. (\%) & 0,180 & 0,180 & 0,180 & 0,180 & 0,180 & 0,125 \\
\hline Sódio & 0,230 & 0,230 & 0,230 & 0,230 & 0,230 & 0,220 \\
\hline
\end{tabular}

1 - Ração formulada para suínos castrados cirurgicamente.

2 - Núcleo para suínos em terminação. Composição: ácido fólico (mín. 6,00 mg/kg), ácido pantoténico (mín. $200.00 \mathrm{mg} / \mathrm{kg}$ ), bacitracina de zinco (mín. 1.375,00 mg/kg), biotina (mín. 1,00 mg/kg), cálcio (mín. 175,00 g/kg), cálcio (máx. 220,00 g/kg), cobalto (mín. 11,50mg/kg), cobre (mín. 4.080,00 mg/kg), ferro (mín. 2.500,00 mg/kg), fitase (mín. 12,50 ftu/kg), fluor (máx.200.00mg/kg), fósforo (mín. 46.309/kg), iodo (mín. 25,00 mg/kg), manganês (mín 1750,00 mg/kg), niacina (mín. 440.00mg/kg), selênio (mín 10,00 mg/kg), sódio (mín 47.somg/kg), vitamina a (mín. 105.000,00 ui) kg, vitamina b1 (mín. 20,o0mg/kg), vitamina bi2 (mín. $300.00 \mathrm{mcg} / \mathrm{kg}$ ), vitamina b2 (mín.90,00 mg/kg), vitaminab6 (mín. 20,0omg/kg), vitaminad3 (mín.20.000.00 ui/kg), vitamina e (mín. 240,00 ui/kg), vitamina k3 (mín. 30,00 mg/kg), zinco(mín. 2.500,00 mg/kg). 
foi realizado seguindo as normas preconizadas pelo Ministério da Agricultura, Pecuária e Abastecimento (MAPA).

Após o período de resfriamento, as amostras de lombo suíno (músculo Longissimus dorsi) foram descongeladas em geladeira convencional em temperatura média de $4^{\circ} \mathrm{C}$ por um período de 48 horas. As amostras foram identificadas (IC imunocastrados; CC - castrados cirurgicamente) e em seguida cortadas paralelamente às fibras musculares em amostras iguais com formato cúbico de 2,5 cm. Seguida esta fase, as amostras foram alocadas em béquer pré-aquecido em banho-maria (equipamento SOLAB SL-150) onde ficaram por tempo estabelecido de 20 minutos para pré-cozimento até atingirem a temperatura em seu centro geométrico de $70^{\circ} \mathrm{C}$.

Após cozimento, as amostras foram alocadas em copos plásticos identificados em códigos numéricos de três dígitos. As amostras de número 587 e 246 foram estabelecidas para animais castrados cirurgicamente e 894 e 358 foram estabelecidas para animais imunocastrados. Para a avaliação sensorial do odor das carnes, as amostras foram servidas de modo sequencial em cabines individuais.

Trinta provadores não treinados assinaram o termo de consentimento e realizaram a análise do odor das amostras, preenchendo formulário de avaliação. Os julgadores foram desafiados através do teste de poder discriminativo do tipo triangular (teste de diferença).

A distribuição triangular das amostras (CC, CC e IM; IM, IM e CC) objetivou desafiar o poder discriminativo dos julgadores em averiguar a diferença entre odor na carne de animais imunocastrados ou castrados cirurgicamente, identificando no formulário qual das amostras apresentava (ou se não apresentava) odor diferenciado. Foram utilizadas amostras de carne de suínos CC e IM com nível de energia metabolizável (EM) na ração consumida de $3230 \mathrm{Kcal} / \mathrm{Kg}$, no intuito de evitar a influência da alimentação no odor da carne.

Os dados médios obtidos nos diferentes níveis de energia (tratamento 1 ao tratamento 5) foram submetidos à análise de variância (teste F) e análise de regressão linear em nível de 5 e 1\% de significância. Não houve análise de regressão no período $B$ em caso de efeito significativo no período A para evitar o efeito residual. Os tratamentos T3a (IM) e T3b (CC) foram comparados pelo teste de $\mathrm{F}$ a nível de $5 \%$ de significância. A análise foi realizada com o auxílio do programa de análises estatísticas SAEG (1998).

\section{RESULTADOS E DISCUSSÃO}

O consumo de ração foi avaliado no período que correspondente ao intervalo da primeira aplicação da vacina à segunda aplicação, no período referente ao intervalo entre 
a segunda imunização ao abate e no período total experimental na tentativa de uma observação completa do comportamento alimentar em função da imunocastração. Entre a primeira e a segunda aplicação da vacina, esperava-se um consumo característico de animais inteiros, ou seja, maior interação entre os animais e um menor consumo comparado ao período pós-imunização. Após a segunda imunização, esperava-se um consumo mais acentuado de ração, com um maior ganho de peso (Tabela 2). Esses dois comportamentos já foram observados em várias pesquisas (Fontes et al., 2012; Dunshea et al., 2001).

O efeito do nível de energia metabolizável sobre o consumo de ração diário no período total, ganho de peso diário no período B e ganho de peso diário no período total pode ser observado na Figura 1. Não foi observado $(P>0,05)$ efeito dos níveis de energia metabolizável sobre o consumo de ração diário nos períodos $A$ e $B$. No período total $(T)$ o consumo de ração aumentou linearmente $\left(y=0,0003 x+1,637 x ; R^{2}=0,76\right)$ com o nível de energia $(P<0,05)$. Esse resultado contradiz a teoria de regulação de consumo pela energia, onde se esperava um decréscimo no consumo na medida em que se aumentassem os níveis de EM nos tratamentos. Como os animais iniciaram a fase experimental com peso médio de $85 \mathrm{~kg}$, peso acima do proposto para a fase de terminação $(70 \mathrm{Kg})$, terminando essa fase também com um peso maior ao indicado na tabela $(100 \mathrm{Kg})$, a exigência de mantença e consequentemente a exigência em energia desses animais pode ter sido mais elevada.

A exigência em energia para mantença é a quantidade de energia necessária, oriunda do alimento, que não resultará em perda ou ganho de energia corporal. No entanto, Chwalibog (1991) definiu a exigência energética de mantença como aquela necessária para manter o equilíbrio entre o turnover proteico e de gordura, mantendo também o equilíbrio entre as atividades normais de locomoção e temperatura corporal. De acordo com Sakomoura e Rostagno (2007), essa definição é diferente da clássica sobre a exigência de energia de mantença, levando em conta que o equilíbrio entre o turnover de proteína e gordura ocorre quando se tem retenção negativa de gordura e retenção positiva de proteína.

Não se observou efeito do nível de energia sobre o consumo de ração nos períodos A e B (CDA e CDB, respectivamente). Corroborando, Zamaratskaia et al. (2009), observaram que o consumo diário de ingestão de ração em machos inteiros após a segunda imunização aumenta consideravelmente, o que pode estar relacionado com a queda abrupta dos níveis de testosterona após a imunocastração. Silva et al. (1998), trabalhando com níveis de energia digestível para suínos machos inteiros dos 60 aos 100 
quilos de peso vivo, não observaram diferenças significativas no consumo de ração médio diário enquanto se aumentavam os níveis de energia digestível na dieta.

Tabela 2 - Valores médios por período (A, B ou T), de consumo diário (CD), ganho de peso diário (GPD), conversão alimentar (CA), consumo diário de energia (CDE) e consumo diário de lisina $(\mathrm{CDL})$ para animais imunocastrados.

\begin{tabular}{|c|c|c|c|c|c|c|}
\hline \multirow[b]{2}{*}{ Período ${ }^{\star 3}$} & \multicolumn{6}{|c|}{ Nível de energia metabolizável (kcal/kg) } \\
\hline & 3030 & 3130 & 3230 & 3330 & 3430 & CV (\%) \\
\hline & \multicolumn{6}{|c|}{ Consumo de ração (CD) (Kg/dia) } \\
\hline$A$ & 2,763 & 2,735 & 2,788 & 3,033 & 2,848 & 6,133 \\
\hline B & 2,575 & 2,552 & 2,586 & 2,527 & 2,623 & 8,644 \\
\hline \multirow[t]{2}{*}{$T^{\star 1}$} & 2,672 & 2,627 & 2,691 & 2,751 & 2,775 & 3,417 \\
\hline & \multicolumn{6}{|c|}{ Ganho de peso (GPD) (Kg/dia) } \\
\hline A & 0,845 & 0,787 & 0,908 & 0,960 & 0,940 & 17,392 \\
\hline$B^{\star 1}$ & 0,845 & 0,787 & 0,908 & 0,960 & 0,940 & 12,438 \\
\hline \multirow[t]{2}{*}{$T^{\star 1}$} & 1,025 & 0,967 & 1,012 & 1,098 & 1,119 & 7,775 \\
\hline & \multicolumn{6}{|c|}{ Conversão alimentar (CA) (Kg/Kg) } \\
\hline A & 2,322 & 2,245 & 2,549 & 2,498 & 2,217 & 14,313 \\
\hline B & 3,048 & 3,256 & 2,900 & 2,666 & 2,867 & 13,133 \\
\hline \multirow[t]{2}{*}{$\mathrm{T}$} & 2,615 & 2,733 & 2,704 & 2,518 & 2,485 & 8,945 \\
\hline & \multicolumn{6}{|c|}{ Consumo de energia metabolizável (CDE) (Kcal/dia) } \\
\hline$A^{\star 2}$ & 8371,89 & 8560,55 & 9005,24 & 10099,89 & 9768,64 & 6,341 \\
\hline B & 7802,25 & 7987,76 & 8352,78 & 8414,91 & 8996,89 & 8,660 \\
\hline \multirow[t]{2}{*}{$T^{\star 2}$} & 8096,16 & 8222,51 & 8691,93 & 9160,83 & 9518,25 & 3,414 \\
\hline & \multicolumn{6}{|c|}{ Consumo de lisina (CDL) (g/dia) } \\
\hline$A^{\star 1}$ & 28,727 & 28,261 & 29,474 & 31,239 & 30,215 & 5,820 \\
\hline B & 25,746 & 25,522 & 25,857 & 25,273 & 26,226 & 8,644 \\
\hline$T^{\star 2}$ & 26,720 & 26,269 & 26,906 & 27,506 & 28,415 & 4,076 \\
\hline
\end{tabular}

${ }^{* 1}$ Efeito Linear $(P<0,05)$.

*2 Efeito Linear $(P<0,01)$.

*3Período A - correspondente ao intervalo da primeira aplicação da vacina à segunda aplicação; Período B - referente ao intervalo entre a segunda imunização ao abate; Período T - caracterizando o período total experimental. 


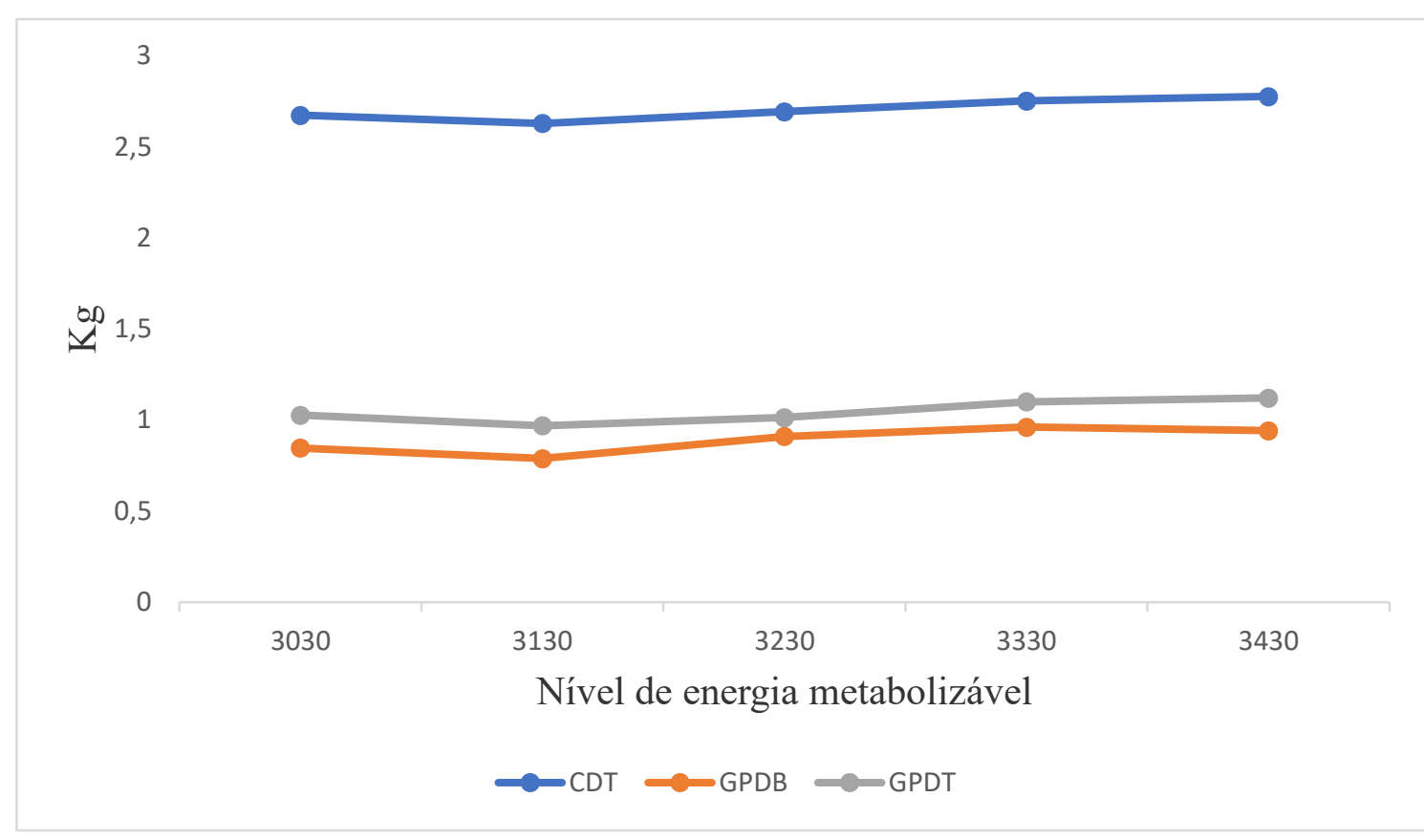

Figura 1 - Gráfico do Consumo de Ração Diário no Período Total (CDT), Ganho de Peso Diário no Período B (GPDB) e Ganho de Peso Diário Total (GPDT) de suínos imunocastrados em função dos níveis de EM (Kcal/Kg). ( $y=0,0003 x+1,637 x ; R 2=$ $0,76)$.

Em um estudo utilizando animais inteiros, imunocastrados e castrados cirurgicamente, Zeng et al. (2002), utilizando quatro tratamentos comparando dois níveis energéticos na dieta definidos como de alta energia (9,7 MJ, aproximadamente 2317 Kcal) e de baixa energia (8,3 MJ, aproximadamente $1982 \mathrm{Kcal})$, e em duas fases de produção (crescimento e terminação), observaram que os animais inteiros antes da segunda imunização (de 10 a 17 semanas de idade) se alimentaram menos do que animais castrados cirurgicamente em ambas as dietas, com os animais imunocastrados em um nível intermediário de consumo. Após a segunda imunização (17 semanas), o comportamento se manteve, com os animais inteiros se alimentando menos que castrados cirurgicamente e animais imunocastrados em um nível intermediário. Os animais inteiros continuaram se alimentando menos, porém os animais imunocastrados apresentaram consumo similar aos castrados cirurgicamente.

Houve efeito $(P<0,05)$ sobre o ganho de peso diário no período $B(y=0,0004 x-$ $\left.0,2845, R^{2}=0,65\right)$ e o ganho de peso diário total $\left(y=0,0003 x+0,0122, R^{2}=0,64\right)$. Não foi observado efeito no período $A$. Esses resultados foram semelhantes aos encontrados por Silva et al. (1998), que utilizaram níveis de ED variando de 3200 a $3700 \mathrm{kcal} / \mathrm{kg}$ para suínos machos inteiros dos 60 aos $100 \mathrm{~kg}$. 


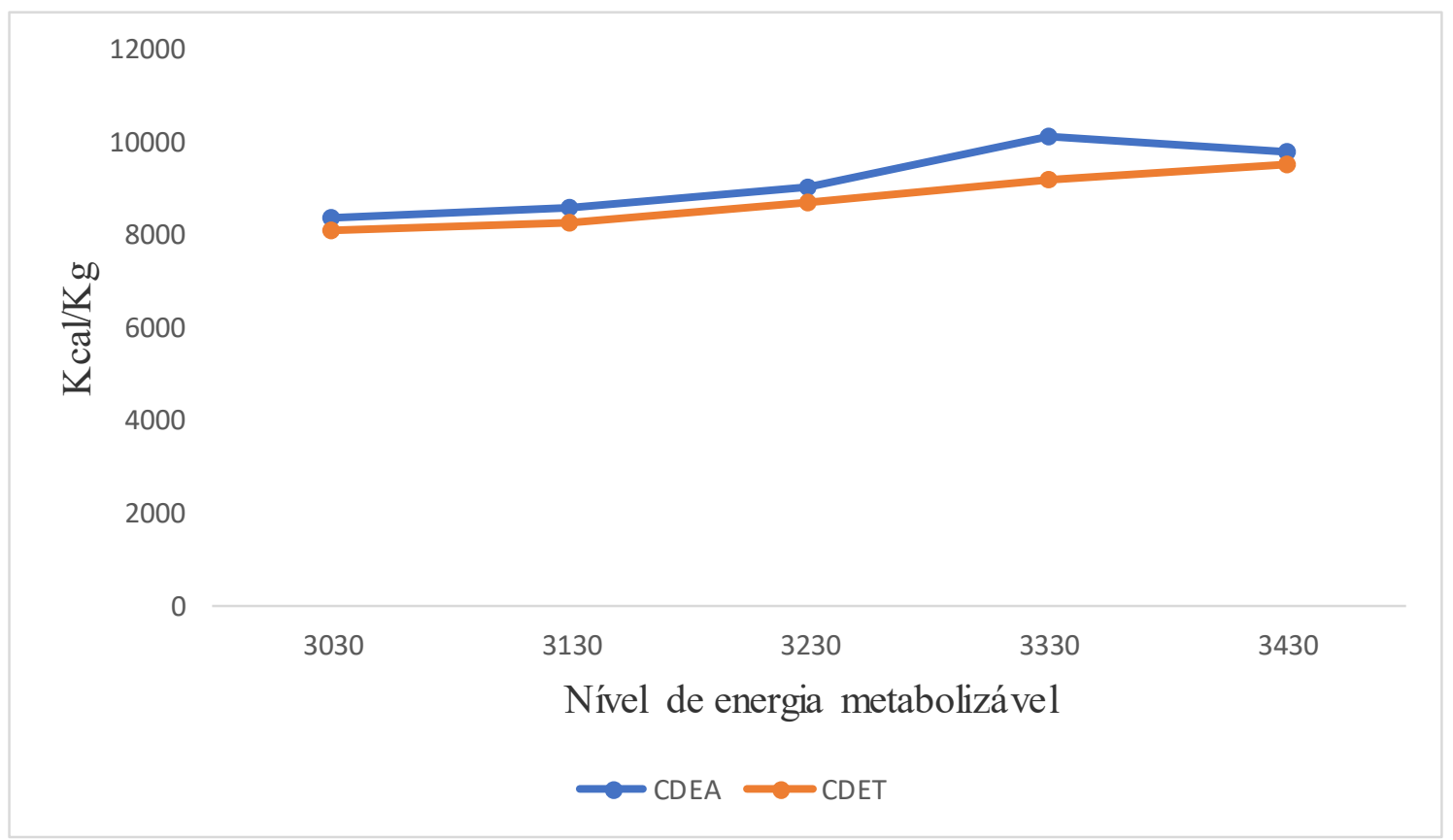

Apesar dos animais apresentarem resposta positiva no consumo de ração diário e no ganho de peso diário com o aumento da EM nos tratamentos, não se observou efeito significativo na conversão alimentar em nenhum dos períodos. Os suínos em crescimento e terminação recebendo ração têm seu consumo e sua conversão alimentar dependentes em grande parte de seu consumo de energia (Rostagno et al., 2017), o que foi observado neste trabalho. O consumo diário energia (CDE) acompanhou o aumento do nível de energia nos tratamentos no período A e período total como representado na Figura 2.

Foi observado efeito linear crescente $(P<0,01)$ para CDEA e CDET $(y=0,0043 x-$ $4,844, R^{2}=0,84$; e y $=0,0038 x-3,4804, R^{2}=0,97$ respectivamente).

A recomendação de Rostagno et al. (2017) para consumo de ração para suínos inteiros dos 111 aos 134 dias de idade, com alto potencial genético e desempenho médio é, em média, 2,71 Kg/dia, para uma dieta com $3230 \mathrm{Kcal} / \mathrm{Kg}$ de energia metabolizável, o que significa um consumo de energia diário de aproximadamente 8750 $\mathrm{Kcal} / \mathrm{kg}$, ligeiramente superior ao observado no experimento para o mesmo nível energético em período total, que foi de $8691,93 \mathrm{Kcal} / \mathrm{Kg}$.

Figura 2 - Consumo Diário de Energia em A (CDEA) e Consumo Diário de Energia Total (CDET) de suínos imunocastrados em função dos níveis de EM (Kcal/Kg; y = $0,0043 x-4,844, R^{2}=0,84$; e $y=0,0038 x-3,4804, R^{2}=0,97$, respectivamente para CDEA e CDET).

Em contrapartida, o consumo de ração em $\mathrm{Kg} /$ dia preconizado para suínos machos castrados de alto potencial genético e desempenho médio, utilizando o mesmo 
nível de EM, com animais de 113 a 140 dias de idade, de 70 a $100 \mathrm{~kg}$ de peso vivo é de 3,027, totalizando um consumo diário de energia de $9780 \mathrm{Kcal} / \mathrm{Kg}$, consumo superior tanto em relação à $\operatorname{CDEA}(9005,24 \mathrm{Kcal} / \mathrm{Kg})$ quanto a $\operatorname{CDEB}(8352,78)$, etapa em que se esperava obter um comportamento alimentar semelhante aos castrados cirurgicamente.

Considerando os valores apresentados na Tabela Brasileira de Aves e Suínos (2017), as exigências de consumo de ração e energia metabolizável para animais inteiros param aos 147 dias de idade, e adotarmos, para o período B, o exigido para animais castrados dos 141 aos 160 dias de idade, essa diferença torna-se mais discrepante. O recomendado para animais castrados nessa faixa etária e com peso vivo de 100 a $120 \mathrm{~kg}$ é de 3,399 Kg/dia de ração e $10978 \mathrm{Kcal} / \mathrm{kg}$ de EM.

Mesmo que no período $A$ os animais tenham apresentado CDE superior em todos os tratamentos comparados com o período $B$, o valor está próximo da exigência de consumo de energia metabolizável em Kcal/dia (9210), preconizado nas Tabelas Brasileiras para animais na mesma fase.

$O$ consumo de EM observado no experimento foi inferior $(P<0,01)$ em relação às exigências de produção sugerindo que os animais regularam seu consumo não só pela energia. A regulação do consumo é resultante da ingestão de alimentos de composições variáveis em valores energéticos ou composição de nutrientes. A presença de subprodutos oriundos da quebra de proteínas ou lipídeos no trato gastrointestinal pode, direta ou indiretamente, via hormônios, estimular os receptores vagais promovendo impulsos diretamente no cérebro. O cérebro recebe um grande número de sinais para a ingestão de alimento, cujas informações habilitam o mesmo a realizar um mecanismo de controle alimentar, direcionando os animais a realizar "escolhas alimentares" apropriadas, sejam elas de caráter quantitativo (energia) ou de caráter qualitativo (macro e micronutrientes, proteínas e aminoácidos; Anderson et al., 1984).

O CDL apresentou efeito linear positivo dos níveis de energia nos períodos $A(y=$ $\left.0,006 x+10,349 ; R^{2}=0,81\right),(P<0,05)$ e $T\left(y=0,0046 x+12,215 ; R^{2}=0,91\right),(P<0,01)$, (Figura 3).

Esse aumento no consumo de lisina se deve ao aumento no consumo diário de ração, já que o percentual de lisina digestível dietético se manteve constante em todos os tratamentos (1,00\%). A recomendação média para consumo diário de lisina digestível para suínos inteiros com 111 dias de idade até 134 dias e com 70 a 100 kg de peso vivo aproximadamente, em uma dieta com $3230 \mathrm{Kcal} / \mathrm{Kg}$ de energia metabolizável é de 27,10 g/dia, semelhante ao observado no experimento, que foi de $26,91 \mathrm{~g} / \mathrm{dia}$ para o tratamento com $3230 \mathrm{Kcal} / \mathrm{Kg}$. Vale ressaltar que os animais deste estudo se enquadram em uma faixa de idade (em dias) maior do que o limite recomendado na Tabela Brasileira 
de Aves e Suínos (2017). Os animais mais velhos são menos exigentes em proteína. Portanto, é plausível considerar o consumo de $26,9 \mathrm{~g} /$ dia se encontra nos padrões aceitáveis.

A relação lisina digestível/energia metabolizável para a mesma categoria animal, com a mesma idade é de 0,114\%/Mcal. A relação observada no experimento foi de 0,115\%/Mcal, estando, portanto, de acordo com o preconizado.

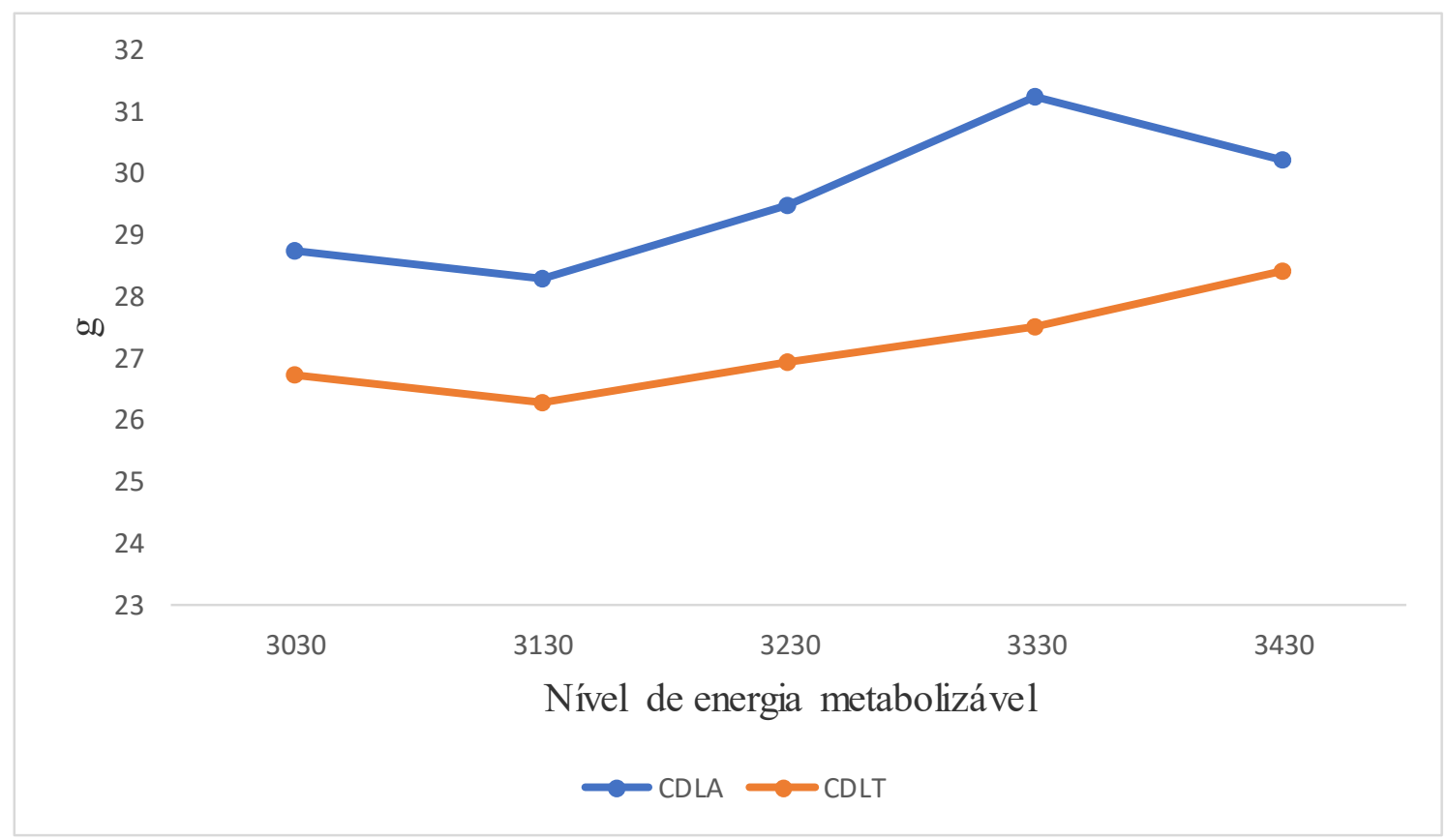

Figura 3 - Consumo de Lisina Diário no Período A (CDLA) e Consumo de Lisina Diário Total (CDLT) de suínos imunocastrados em função dos níveis de EM (Kcal/Kg). $\quad\left(y=0,006 x+10,349, R^{2}=0,81\right.$ e $y=0,0046 x+12,215, R^{2}=0,91$, $\begin{array}{lllll}\text { respectivamente } & \text { para } & \text { e }\end{array}$

Outro fator que pode interferir no consumo alimentar é a palatabilidade do alimento ingerido. O óleo de soja é reconhecidamente um dentre os vários ingredientes utilizados na dieta animal que melhoram a palatabilidade da ração. Como as dietas obtiveram níveis crescentes de óleo de soja objetivando-se estabilizar os níveis preconizados de energia metabolizável, a diferença de utilização do óleo na primeira dieta (3030 Kcal/Kg de EM, tratamento 1) à última (3430 Kcal/Kg, tratamento 5) foi de 5,3 Kg de óleo para cada 100 quilos de ração. O nível crescente de inclusão de óleo nas formulações pode ter estimulado o consumo de ração. Portanto, existe grande possibilidade do consumo de ração diário ter sido influenciado pela palatabilidade à medida em que se aumentavam os níveis de EM dietéticos. 
Os animais no período $\mathrm{A}$ apresentaram valores absolutos inferiores em todos os níveis de EM comparados ao período $B$. Essa diferença, mesmo não significativa, sugere melhor eficiência de conversão energética de animais inteiros ou imunocastrados antes da segunda imunização.

Tabela 3 - Conversão calórica e conversão de lisina de animais imunocastrados com diferentes níveis de EM.

\begin{tabular}{|c|c|c|c|c|c|c|}
\hline \multirow[b]{2}{*}{ Período*2 } & \multicolumn{6}{|c|}{ Nível de energia metabolizável (kcal/kg) } \\
\hline & 3030 & 3130 & 3230 & 3330 & 3430 & CV (\%) \\
\hline & \multicolumn{6}{|c|}{ Conversão calórica (Mcal/Kg) ${ }^{* 1}$} \\
\hline A & 7,04 & 7,03 & 8,23 & 8,23 & 7,60 & 14,30 \\
\hline B & 9,23 & 10,19 & 9,37 & 8,88 & 9,83 & 13,40 \\
\hline \multirow[t]{2}{*}{$\mathrm{T}$} & 7,92 & 8,55 & 8,73 & 8,38 & 8,52 & 8,40 \\
\hline & \multicolumn{6}{|c|}{ Conversão de lisina $(\mathrm{g} / \mathrm{Kg})^{\star 1}$} \\
\hline$A$ & 24,47 & 23,42 & 26,94 & 25,68 & 23,84 & 14,20 \\
\hline B & 30,48 & 32,76 & 29,00 & 26,66 & 28,66 & 13,30 \\
\hline $\mathrm{T}$ & 26,15 & 27,33 & 27,04 & 25,18 & 25,44 & 7,50 \\
\hline
\end{tabular}

${ }^{*}$ Não houve efeito linear em nível de $5 \%$ de significância $(P=0,067$ e $P=0,062$, respectivamente para conversão calórica e de lisina);

${ }^{* 2}$ Período A - correspondente ao intervalo da primeira aplicação da vacina à segunda aplicação; Período B referente ao intervalo entre a segunda imunização ao abate; Período $\mathrm{T}$ - caracterizando o período total experimental.

A relação energia metabolizável/lisina digestível foi de 0,29; 0,30;0,31;0,32 e 0,32 $\mathrm{Mcal} / \mathrm{g}$ para os tratamentos 1, 2, 3, 4 e 5, respectivamente.

Realizando uma comparação entre os animais CC e os IM com mesmo nível de EM (Tabela 4), em relação ao consumo de ração, ganho de peso, conversão alimentar e consumo de EM, não se observou diferença significativa em nenhum dos parâmetros.

As características de carcaça dos animais IM se encontram na Tabela 5. Em relação às características de carcaça avaliadas, não se observou diferença $(P>0,05)$ entre os tratamentos.

De acordo com a Tabela da ABNT, NBR 12995 (1993), o total de 16 acertos representa diferença $(P<0,05)$, o que significa que os avaliadores não treinados perceberam diferenças nas amostras, porém essa diferença no odor não foi caracterizada como o boar taint, ou seja, não se identificou odor de fezes e nem de urina nas amostras.

Os acertos e erros dos trinta julgadores não treinados na análise sensorial de amostras do músculo Longissimus dorsi se encontram na Tabela 6. 
Tabela 4 - Desempenho de suínos castrados cirurgicamente e imunocastrados alimentados com ração com nível de $3230 \mathrm{Kcal}$ de EM/Kg.

\begin{tabular}{|c|c|c|c|c|}
\hline \multirow{2}{*}{ Período $^{* 1}$} & \multicolumn{3}{|c|}{ Categoria } & \multirow{2}{*}{ Valor $\mathbf{P}$} \\
\hline & Imunocastrados & Castrados Cirurgicamente & CV (\%) & \\
\hline \multicolumn{5}{|c|}{ Consumo de ração (Kg/dia) } \\
\hline$A$ & 2,79 & 2,99 & 6,21 & 0,330 \\
\hline B & 2,59 & 2,54 & 5,13 & 0,883 \\
\hline $\mathrm{T}$ & 2,69 & 2,77 & 3,61 & 0,885 \\
\hline \multicolumn{5}{|c|}{ Ganho de peso (Kg/dia) } \\
\hline$A$ & 1,11 & 1,05 & 15,69 & 0,854 \\
\hline B & 0,91 & 1,02 & 14,35 & 0,362 \\
\hline $\mathrm{T}$ & 1,01 & 1,04 & 13,45 & 0,233 \\
\hline \multicolumn{5}{|c|}{ Conversão alimentar $(\mathrm{Kg} / \mathrm{Kg})$} \\
\hline A & 2,55 & 2,92 & 13,96 & 0,588 \\
\hline B & 2,90 & 2,50 & 17,17 & 0,309 \\
\hline $\mathrm{T}$ & 2,70 & 2,68 & 14,38 & 0,657 \\
\hline \multicolumn{5}{|c|}{ Consumo de EM (Mcal/dia) } \\
\hline$A$ & 9,00 & 9,64 & 6,20 & 0,610 \\
\hline B & 8,35 & 8,19 & 5,13 & 0,609 \\
\hline $\mathrm{T}$ & 8,69 & 8,95 & 3,60 & 0,743 \\
\hline
\end{tabular}

${ }^{*}$ Período A - correspondente ao intervalo da primeira aplicação da vacina à segunda aplicação; Período B referente ao intervalo entre a segunda imunização ao abate; Período $\mathrm{T}$ - caracterizando o período total experimental.

Na análise sensorial, 16 dos 30 avaliadores reconheceram a amostra de animais imunocastrados sendo a "amostra diferente", enquanto 12 reconheceram os animais castrados cirurgicamente e 2 avaliadores não perceberam diferença.

Dentre as 15 amostras de animais IC, cinco pessoas notaram odor mais suave, três notaram odor mais forte e outras sete pessoas notaram diferença, mas não souberam responder de forma precisa. Das 15 amostras de animais CC, quatro notaram odor mais suave, enquanto dois notaram odor mais forte. Outras nove pessoas notaram diferença, mas não souberam identificar com precisão. 
Tabela 5 - Características de carcaça dos animais imunocastrados.

\begin{tabular}{|c|c|c|c|c|c|c|c|}
\hline \multirow{2}{*}{ Variável } & \multicolumn{6}{|c|}{ Nível de energia metabolizável (kcal/kg) } & \multirow{2}{*}{$\begin{array}{c}\text { Valor } \\
\text { P }\end{array}$} \\
\hline & 3030 & 3130 & 3230 & 3330 & 3430 & CV (\%) & \\
\hline Peso de carcaça $(\mathrm{Kg})$ & 116,35 & 112,15 & 109,90 & 107,00 & 116,70 & 8,46 & 0,965 \\
\hline $\begin{array}{l}\text { Espessura de toucinho } \\
\text { quente }(\mathrm{cm})\end{array}$ & 2,27 & 2,40 & 2,22 & 2,20 & 2,07 & 22,70 & 0,917 \\
\hline $\begin{array}{l}\text { Espessura de toucinho } \\
\text { resfriada }(\mathrm{cm})\end{array}$ & 2,07 & 2,07 & 1,75 & 2,00 & 2,30 & 20,40 & 0,158 \\
\hline $\begin{array}{l}\text { Comprimento de carcaça } \\
\text { (m) }\end{array}$ & 1,20 & 1,18 & 1,16 & 1,23 & 1,19 & 5,07 & 0,061 \\
\hline $\begin{array}{l}\text { Área de olho de lombo } \\
\left(\mathrm{mm}^{2}\right)\end{array}$ & 50,50 & 51,00 & 43,00 & 50,75 & 48,50 & 11,50 & 0,539 \\
\hline
\end{tabular}

Tabela 6 - Acertos e erros dos julgadores não treinados no teste triangular para a determinação de diferenças na carne de suínos imunocastrados (IM) e castrados cirurgicamente (CC).

\begin{tabular}{|c|c|c|c|c|c|}
\hline Julgador & Acerto & Erro & Julgador & Acerto & Erro \\
\hline 1 & $\mathrm{IM}$ & & 16 & CC & \\
\hline 2 & & $x$ & 17 & $\mathrm{IM}$ & \\
\hline 3 & $\mathrm{IM}$ & & 18 & & $x$ \\
\hline 4 & $\mathrm{CC}$ & & 19 & CC & \\
\hline 5 & $\mathrm{IM}$ & & 20 & & $x$ \\
\hline 6 & & $x$ & 21 & CC & \\
\hline 7 & $\mathrm{IM}$ & & 22 & & $x$ \\
\hline 8 & & $x$ & 23 & & $x$ \\
\hline 9 & $\mathrm{IM}$ & & 24 & $\mathrm{IM}$ & \\
\hline 10 & & $x$ & 25 & & $x$ \\
\hline 11 & IM & & 26 & & $x$ \\
\hline 12 & & $x$ & 27 & $C C$ & \\
\hline 13 & & $x$ & 28 & & $x$ \\
\hline 14 & $\mathrm{CC}$ & & 29 & & $x$ \\
\hline 15 & $\mathrm{IM}$ & & 30 & $\mathrm{IM}$ & \\
\hline Total & 9 & 6 & & 7 & 8 \\
\hline
\end{tabular}




\section{CONCLUSÃO}

Não foi possível estimar o melhor nível de energia metabolizável para suínos imunocastrados. O ganho de peso foi maior nos animais que receberam níveis mais elevados de EM. Dentre os tratamentos utilizados, os animais com $3430 \mathrm{Kcal} / \mathrm{Kg}$ de EM obtiveram melhore resultados com relação a ganho de peso diário, consumo de energia metabolizável e consumo de lisina diária. Os níveis de EM, pré e pós imunização não influenciam a qualidade das carcaças de suínos.

\section{Notas informativas}

O uso de animais foi aprovado pela CEUA (Comissão de Ética sobre uso de animais) com registro 037/13.

\section{REFERENCIAS}

ANDERSON, G.H.; LI, E.T.S.; GLANVILLE, N.T. Brain mechanisms and the quantitative and qualitative aspects of food intake. Brain Research Bulletin, v.12, p.167-173, 1984.

BOGONI, D.A.; ANDRETTA, I.; KIPPER, M. et al. Produção de suínos machos em crescimento: uma meta-análise. Revista Brasileira de Saúde e Produção Animal, Salvador, v.16, n.1, p.130-138, 2015.

CHWALIBOG, A. Factorial Estimation of Energy Requirement for Egg Production. Poltry Science, v.71, p.509-515, 1991.

DUNSHEA, F.R.; COLANTONI, C.; HOWARD, K. et al. Vaccination of boars with a GnRH vaccine (Improvac $(\mathbb{R})$ eliminates boar taint and increases growth performance. Journal of Animal Science, v.79, p.2524-2535, 2001.

FISCHER, J.; GERLACH, C.; MEIER-DINKEL, L. et al. 2-Aminoacetophenone - A hepatic skatole metabolite as a potential contributor to boar taint. Food Research International, v.62, p.35-42, 2014.

FONTES, D.O; SOUZA, L.P.O; ROSA, B.O. Ajustes nutricionais em dietas para suínos imunocastrados. In: IV SIMPÓSIO MINEIRO DE SUINOCULTURA., 2012, Lavras, Anais...

LERVIK, S.; OSKAM, I.; KROGENAES, A. et al. Androsterone and testosterone levels and testicular morphology of Duroc boars related to estimated breeding value for androsterone. Theriogenology, v.79, p.986-994, 2013.

LOPES, K.R.F; SILVA, A.R. Castração química de mamíferos machos: revisão. Revista Brasileira de Reprodução Animal, Belo Horizonte, v.38, n.1, p.49-53, 2014.

PAULY, C.; SPRING, P.; O'DOHERTY, J.V. et al. Performances, meat quality, and boar taint of castrates and entire male pigs fed a standard and a raw potato starch-enriched diet. Animal, v.2, p.1707-1715, 2008. 
ROSTAGNO, H.S.; ALBINO, L.F.T.; HANNAS, M.I. et al. Tabelas Brasileiras Para aves e suínos; composição de alimentos e exigências nutricionais. Viçosa, MG: Universidade Federal de Viçosa, 2017. 488 p.

SAKOMOURA, N.K; ROSTAGNO, H.S. Métodos de pesquisa em nutrição de monogástricos. Jaboticabal: Funep, 2007.

SILVA, F.C.O.; DONZELE, J.L.; FREITAS, R.T.F. et al. Níveis de Energia Digestível para Suínos Machos Inteiros dos 60 aos 100 kg. Revista Brasileira de Zootecnia, v.27, n.5, p.959-964, 1998.

ZAMARATSKAIA, G.; SQUIRES, E.J. Biochemical, nutritional and genetic effects on boar taint in entire male pigs. Animal, v.3, p.1508-1521, 2009.

ZENG, X.Y.; TURKSTRA, J.A.; JONGBLOED, A.W. et al. Performance and hormone levels of immunocastrated, surgically castrated and intact male pigs fed ad libitum high - and lowenergy diets. Livestock Production Science, v.77, p.1-11, 2002. 